\title{
Aprender y enseñar en entornos virtuales: actividad conjunta, ayuda pedagógica y construcción del conocimiento
}

\author{
Learning and teaching in virtual environments: \\ joint activity, teacher assistance and knowledge construction \\ Javier Onrubia \\ Departamento de Psicología Evolutiva y de la Educación. Universidad de Barcelona. España. \\ javier.onrubia@ub.edu
}

\begin{abstract}
Resumen
El artículo se plantea dos objetivos básicos. El primero es esbozar un marco teórico de carácter constructivista y socio-cultural para el estudio y análisis de los procesos virtuales de enseñanza y aprendizaje, estructurado en torno a los tres conceptos a los que hace referencia el título del texto: "actividad conjunta", "ayuda pedagógica" y "construcción del conocimiento". El segundo es explorar algunas de las implicaciones de este marco para el diseño y evaluación de entornos virtuales de enseñanza y aprendizaje, en general, y para el diseño y evaluación de “objetos de aprendizaje”, en particular.
\end{abstract}

\section{Key words}

Constructivismo de orientación socio-cultural, diseño instruccional, entornos virtuales de enseñanza y aprendizaje, objetos de aprendizaje, prácticas educativas mediadas por las TIC.

\begin{abstract}
The paper proposes a theoretical approach to the learning and teaching processes in virtual environments based on the principles of socio-cultural constructivism. Three key ideas are discussed: learning in virtual environments as a process of knowledge construction, teaching in virtual environments as a process of continuous and sensible assistance to knowledge construction, and teacher-students joint activity as the basic unit of analysis of learning and teaching processes in virtual environments. Implications of these ideas for the design and evaluation of virtual learning environments and learning objects are considered and examined.
\end{abstract}

Key words

Instructional design, ICT-mediated educational practices, learning objects, socioconstructivism, virtual learning environments.

\section{Introducción}

A lo largo de las páginas que siguen, nos proponemos dos objetivos básicos. El primero es esbozar un marco teórico de carácter constructivista y socio-cultural para el estudio y análisis de los procesos virtuales de enseñanza y aprendizaje, articulado en torno a los tres conceptos a los que hace referencia el título del texto: los conceptos de "actividad conjunta", "ayuda pedagógica" y "construcción del conocimiento". El segundo es el de explorar algunas de las implicaciones de este marco para el diseño y evaluación de 
entornos virtuales de enseñanza y aprendizaje, en general, y para el diseño y evaluación de "objetos de aprendizaje", en particular. Este doble objetivo va a dar lugar, igualmente, a la estructura de nuestra exposición. Empezaremos, por tanto, presentando algunas nociones e ideas clave de una visión constructivista y sociocultural de los procesos virtuales de enseñanza y aprendizaje ${ }^{i}$; a continuación, y en la segunda parte del artículo, plantearemos algunas de las reflexiones que esas nociones e ideas pueden sugerir en torno al diseño y evaluación de entornos y procesos virtuales de enseñanza y aprendizaje.

Nuestro planteamiento pretende, a partir de lo dicho y en último término, aportar algunos elementos, de carácter sin duda parcial y hasta cierto punto tentativo, a una tarea a nuestro juicio fundamental y en buena medida pendiente: la elaboración de un marco teórico de referencia sobre los procesos virtuales de enseñanza y aprendizaje, capaz de guiar y orientar tanto las aproximaciones analíticas y explicativas a dichos procesos como las tareas de diseño y evaluación de entornos, materiales y propuestas de enseñanza y aprendizaje virtual. La progresiva elaboración de un marco de estas características, que entendemos debe ser necesariamente multidisciplinar, puede ayudar a prevenir dos riesgos que, en nuestra opinión, han estado y están presentes demasiado a menudo en algunas de las aproximaciones, tanto teóricas como prácticas, que en ocasiones se han desarrollado en este ámbito. El primero de estos riesgos es el de no reconocer y considerar suficientemente la complejidad de las relaciones entre las nuevas tecnologías de la información y la comunicación (TIC) y las prácticas educativas, asumiendo una visión lineal y simplista según la cual la incorporación de las TIC a dichas prácticas constituye, en sí misma y necesariamente, una mejora de la calidad de las mismas. El segundo de dichos riesgos es el de centrar la discusión sobre la incorporación de las TIC a los procesos de enseñanza y aprendizaje en los aspectos tecnológicos más que en los propiamente educativos. El avance hacia un marco teórico multidisciplinar como el que proponemos puede, a nuestro juicio, ayudar a evitar estos riesgos, ayudando a que la incorporación a las prácticas educativas de las nuevas herramientas tecnológicas, y el diseño y desarrollo de procesos virtuales de enseñanza y aprendizaje apoyados en esas herramientas, estén presididos, en los distintos casos, por la necesaria reflexión y por la suficiente fundamentación conceptual.

\section{Aprender $y$ enseñar en entornos virtuales: una perspectiva constructivista y socio-cultural}

\section{El aprendizaje virtual como proceso de construcción}

Caracterizar el aprendizaje en entornos virtuales como un proceso de construcción supone, esencialmente, afirmar que lo que el alumno aprende en un entorno virtual no es simplemente una copia o una reproducción de lo que en ese entorno se le presenta como contenido a aprender, sino una reelaboración de ese contenido mediada por la estructura cognitiva del aprendiz. El aprendizaje virtual, por tanto, no se entiende como una mera traslación o transposición del contenido externo a la mente del alumno, sino como un

Aprender y enseñar en entornos virtuales: Actividad conjunta, ayuda pedagógica y construcción del conocimiento. Javier Onrubia 
proceso de (re)construcción personal de ese contenido que se realiza en función, y a partir, de un amplio conjunto de elementos que conforman la estructura cognitiva del aprendiz: capacidades cognitivas básicas, conocimiento específico de dominio, estrategias de aprendizaje, capacidades metacognitivas y de autorregulación, factores afectivos, motivaciones y metas, representaciones mutuas y expectativas... La actividad mental constructiva que el alumno, al poner en juego este conjunto de elementos, desarrolla en torno al contenido se configura, desde esta perspectiva, como clave fundamental para el aprendizaje, y la calidad de tal actividad mental constructiva, por lo mismo, se configura como clave fundamental para la calidad del aprendizaje: ni toda actividad que el alumno realiza cuando aprende conlleva actividad mental constructiva, ni toda actividad mental constructiva es igualmente deseable ni óptima para un aprendizaje de calidad.

El "postulado constructivista" y la importancia atribuida a la actividad mental constructiva del alumno en su proceso de aprendizaje tienen múltiples e importantes implicaciones para una comprensión más afinada de cómo se aprende en entornos virtuales y de qué se puede hacer desde la enseñanza para promover ese aprendizaje. Nos detendremos muy brevemente en dos de ellas, por su relevancia para nuestra discusión posterior. La primera es la diferencia entre la "estructura lógica" del contenido y la "estructura psicológica" del mismo. La estructura lógica de un contenido remite a la organización interna del material de aprendizaje en sí mismo, y puede considerarse estable entre contextos, situaciones y aprendices. La estructura psicológica del contenido, en cambio, remite a la organización de ese material para un alumno concreto, y depende de de lo que, en cada momento, el alumno aporta al proceso de aprendizaje. Esta diferencia permite distinguir entre dos condiciones, igualmente necesarias pero distintas entre sí, que deben cumplirse para que el alumno pueda atribuir significado al contenido que debe aprender. Por un lado, la significatividad lógica, relacionada con la estructura y organización interna del contenido a aprender. Por otro, la significatividad psicológica, relacionada con el hecho de que el aprendiz disponga de elementos en su estructura cognitiva que pueda poner en relación de manera sustantiva y no arbitraria, de manera profunda y no superficial, con ese contenido. El punto a destacar es que, mientras la significatividad lógica puede garantizarse, esencialmente, desde el diseño del material de aprendizaje, al margen en buena medida de las características concretas de los alumnos a los que ese material se dirige, la significatividad psicológica sólo puede asegurarse mediante formas de ayuda que permitan la adaptación cuidadosa y continuada, en el propio proceso de aprendizaje, de ese material a los alumnos concretos que deben aprenderlo. De ahí la insuficiencia, desde esta perspectiva, de una visión del diseño de los procesos virtuales de enseñanza y aprendizaje virtual centrada únicamente en el diseño de materiales, al margen de las características de los alumnos concretos a los que se dirige y de la dinámica de cambio y evolución de esas características en el contexto particular de la situación de aprendizaje de que se trate.

Aprender y enseñar en entornos virtuales: Actividad conjunta, ayuda pedagógica y construcción del conocimiento. Javier Onrubia 
La segunda implicación del "postulado constructivista" en relación con el aprendizaje virtual que queremos remarcar tiene que ver con el hecho de que lo que el alumno construye y debe construir en un entorno virtual de enseñanza y aprendizaje incluye, al menos, dos tipos distintos de representaciones. Por un lado, representaciones sobre el significado del contenido a aprender. Y por otro, representaciones sobre el sentido que tiene para él aprender ese contenido, sobre los motivos para hacerlo, las necesidades que ese aprendizaje cubre y las consecuencias que supone para la percepción de uno mismo como aprendiz. Ambos tipos de representaciones se construyen, de acuerdo con lo dicho, de manera dinámica, contextual y situada, a partir de lo que aporta en cada momento el aprendiz: ni el significado ni el sentido que el alumno construye están, meramente, en el material que es objeto de aprendizaje, ni su construcción queda asegurada por el diseño de dicho material.

\section{La enseñanza en entornos virtuales como proceso de ayuda}

La actividad mental constructiva desarrollada por el alumno no asegura, necesariamente, una construcción óptima de significados y sentidos en torno al nuevo contenido de aprendizaje. Por un lado, porque el alumno puede no disponer de los recursos cognitivos más adecuados para asimilar el nuevo contenido. Por otro, porque, incluso si los tiene, puede no activarlos, o no establecer las relaciones más significativas y relevantes posible entre esos recursos y el contenido en cuestión. La interacción entre alumno y contenido, por tanto y dicho en otros términos, no garantiza por sí sola formas óptimas de construcción de significados y sentidos.

El elemento que debe tratar de facilitar esas formas óptimas de construcción no es otro que la ayuda educativa ofrecida por el profesor. Esta ayuda debe entenderse, al igual que la propia construcción que realiza el aprendiz, como un proceso, que permita la adaptación dinámica, contextual y situada a la que antes hacíamos referencia entre el contenido a aprender y lo que el alumno puede aportar y aporta a ese aprendizaje en cada momento. Ayudar al aprendizaje virtual, por tanto, no es simplemente una cuestión de presentar información o de plantear tareas a realizar por parte del alumno. Es, esencialmente, seguir de manera continuada el proceso de aprendizaje que éste desarrolla, y ofrecerle los apoyos y soportes que requiera en aquellos momentos en que esos apoyos y soportes sean necesarios. Así entendida, la enseñanza en entornos virtuales tiene un componente necesario de "realización conjunta de tareas" entre profesor y alumno: sólo a partir de esa realización conjunta se podrá realizar una intervención sensible y contingente que facilite realmente al alumno el ir más allá de lo que su interacción solitaria con el contenido le permitiría hacer. De nuevo, ello encaja difícilmente con una visión del diseño de los procesos virtuales de enseñanza y aprendizaje centrada, única o prioritariamente, en el diseño de los contenidos o materiales de aprendizaje.

De acuerdo con esta idea de sensibilidad y contingencia de la ayuda, puede postularse que la ayuda educativa más eficaz en los entornos virtuales de enseñanza y aprendizaje

Aprender y enseñar en entornos virtuales: Actividad conjunta, ayuda pedagógica y construcción del conocimiento. Javier Onrubia 
es la que cumple el principio de "ajuste de la ayuda". Es decir, aquella que incluye apoyos y soportes de carácter diverso; que va cambiando a lo largo del proceso de enseñanza y aprendizaje pero que no lo hace al azar sino a partir de -y en función de- los cambios en la propia actividad mental constructiva desarrollada por el alumno; que "reta" al aprendiz a revisar y profundizar tanto el significado como el sentido que atribuye al nuevo contenido a aprender; que le ofrece instrumentos y "prótesis" para que pueda afrontar y superar esos retos, y que se interesa por promover de manera cada vez mayor la capacidad del alumno para utilizar estratégicamente el conocimiento que va aprendiendo y para seguir aprendiendo de manera cada vez más autónoma y autorregulada. La concreción en detalle de las formas de actuación del profesor que permiten esta ayuda ajustada en entornos virtuales de enseñanza y aprendizaje es, aún, una cuestión abierta y necesitada de una mayor profundización y estudio empírico. El conocimiento disponible sobre los mecanismos de influencia educativa a través de los cuales se concreta el ajuste de la ayuda en los contextos presenciales tradicionales de enseñanza y aprendizaje permite, con todo, plantear algunas primeras ideas al respecto. La primera, que la posibilidad de ajustar la ayuda descansa sobre procesos muy finos, continuados y dinámicos de "ajuste interaccional" entre profesor y alumnos, en buena parte apoyados en determinadas formas de uso del lenguaje como instrumento semiótico para la representación conjunta de significados. La segunda, que estos procesos de ajuste interaccional son complejos y problemáticos, y que su aparición exitosa no puede darse en absoluto por descontada en los encuentros entre profesor, alumnos y contenido de enseñanza y aprendizaje.

\section{Actividad conjunta y procesos de enseñanza y aprendizaje en entornos virtuales}

La importancia atribuida a la ayuda educativa y al principio de ajuste de la ayuda para la comprensión de los procesos de enseñanza y aprendizaje en entornos virtuales aconseja ir más allá de un modelo de análisis y explicación de esos procesos basado únicamente en la interacción entre aprendiz y contenido, y sustituirlo por un modelo más amplio, basado en la relación entre tres elementos: la actividad mental constructiva del alumno que aprende, la ayuda sostenida y continuada del que enseña, y el contenido que es objeto de enseñanza y aprendizaje.

La asunción de este triángulo alumno-profesor-contenidos como unidad básica de análisis de los procesos de enseñanza y aprendizaje en contextos virtuales comporta, al mismo tiempo, considerar la articulación entre las actuaciones de profesor y alumnos en torno al contenido y tareas de enseñanza y aprendizaje, la "actividad conjunta" o "interactividad" como factor explicativo fundamental del aprendizaje en estos contextos y de su calidad (Coll, en prensa). Obviamente, la existencia de una "actividad conjunta" no exige la co-presencia ni en el espacio ni en el tiempo de los participantes en la situación: profesor y alumnos pueden hacer y hacen determinadas cosas conjuntamente aunque lo hagan de manera remota y asíncrona. Dicho de otro modo, lo que hace que la "actividad conjunta" sea efectivamente conjunta no es la co-presencia física de los participantes, sino el hecho de que profesor y alumnos actúan el uno para el otro y entre sí, de manera

Aprender y enseñar en entornos virtuales: Actividad conjunta, ayuda pedagógica y construcción del conocimiento. Javier Onrubia

Página 5 de 14 
que las actuaciones de cada participante sólo se entienden y cobran significado en el marco de, y en referencia a, las actuaciones del resto de participantes. Así por ejemplo, cuando el profesor y los alumnos "conversan" en un foro están, sin duda, implicados en un proceso de actividad conjunta, al igual que cuando el profesor revisa un documento previamente enviado por un alumno, o cuando el alumno estudia un material hipertextual previamente puesto por el profesor en el entorno virtual que comparten.

En los procesos virtuales de enseñanza y aprendizaje, esta actividad conjunta está fuertemente condicionada por, al menos, dos tipos de restricciones y potencialidades. En primer lugar, las que provienen de las características de los recursos tecnológicos que constituyen el entorno virtual. Que el entorno virtual de enseñanza y aprendizaje que se esté usando incluya o no herramientas de trabajo colaborativo, que incorpore herramientas de comunicación únicamente asíncronas o tanto síncronas como asíncronas, que disponga de herramientas de evaluación del aprendizaje de los alumnos de un único tipo o de varios (y los tipos concretos de que se trate), o que permita o no al profesor $-\mathrm{y}$ en qué grado y con qué facilidad- personalizar y adaptar las diversas herramientas disponibles, constituyen, sin duda, elementos cruciales para las posibles formas de organización de la actividad conjunta que profesor y alumnos puedan establecer en ese entorno. El segundo tipo de restricciones y potencialidades es el que proviene del diseño instruccional establecido para el proceso de enseñanza y aprendizaje; es el caso, por ejemplo, de las que se derivan de las características de los contenidos que se incluyen en el diseño, de las características de los materiales en que se apoya la presentación de los contenidos, de las actividades de enseñanza y aprendizaje previstas, o de las actividades de evaluación previstas. Las restricciones y potencialidades derivadas de estos dos factores forman lo que, en conjunto, podemos llamar "diseño tecno-pedagógico" (o "inter-actividad tecno-pedagógica potencial”) de los procesos virtuales de enseñanza y aprendizaje, y pueden actuar en diversas direcciones y con diferentes grados de intensidad, prohibiendo, dificultando, permitiendo, facilitando, promoviendo, obligando... determinadas formas de organizar la actividad conjunta por parte de profesor y alumnos.

El diseño tecno-pedagógico, sin embargo, y pese a condicionar obviamente la actividad conjunta, no la determina completamente. Profesor y alumnos pueden, en efecto, acabar realizando actuaciones no previstas ni promovidas por las herramientas tecnológicas del entorno o por el diseño instruccional, o no realizar lo que las herramientas o el diseño permiten o promueven. Así, un entorno virtual de enseñanza y aprendizaje puede disponer de herramientas de trabajo en equipo para el aprendizaje y la construcción de conocimiento y, en cambio, estas herramientas no ser utilizadas efectivamente por los participantes, o emplearse estrictamente como herramientas de comunicación pero no como herramientas de colaboración. O inversamente, un determinado tipo de actividades puede no estar previsto en el diseño pedagógico pero acabar desplegándose espontáneamente en el desarrollo del proceso de enseñanza y aprendizaje gracias a la

Aprender y enseñar en entornos virtuales: Actividad conjunta, ayuda pedagógica y construcción del conocimiento. Javier Onrubia 
existencia de determinadas herramientas tecnológicas de que los participantes pueden emplear con facilidad; o, incluso, un determinado tipo de actividades puede llegar a aparecer en ausencia de las herramientas tecnológicas más adecuadas para llevarlo a cabo, si los participantes definen usos alternativos de las herramientas que sí están disponibles que puedan dar soporte a ese tipo de actividades. De acuerdo con todo ello, un mismo diseño tecno-pedagógico puede dar lugar a formas concretas de organización de la actividad conjunta diversas, y cambiantes entre momentos, situaciones y alumnos concretos. La comprensión de las interrelaciones entre el diseño tecno-pedagógico y las formas de organización de la actividad conjunta efectivamente desarrolladas por profesor y alumnos, y el análisis de la "interactividad real" así establecida, constituye, por todo ello y desde esta perspectiva, un elemento fundamental para la comprensión de qué aprenden (o no) los alumnos en un entorno virtual de enseñanza y aprendizaje y, sobre todo, por qué (o por qué no) lo aprenden.

\section{Algunas implicaciones para el diseño y evaluación de entornos y objetos virtuales de enseñanza y aprendizaje}

\section{Algunas implicaciones para el diseño de entornos y objetos virtuales de enseñanza y aprendizaje}

Los conceptos e ideas anteriores suponen una llamada de atención sobre la imposible neutralidad pedagógica de los entornos virtuales y los objetos de aprendizaje. Frente a lo que se afirma con cierta asiduidad, ni los entornos tecnológicos de enseñanza y aprendizaje ni los objetos de aprendizaje que se diseñan para su utilización en dichos entornos son ni pueden ser educativamente neutros. En primer lugar, porque un entorno o un objeto de aprendizaje siempre incluye restricciones y potencialidades tecnológicas determinadas (una cierta "inter-actividad tecnológica potencial"), que impiden, dificultan, permiten o promueven -entre otras posibilidades- la realización de determinadas actuaciones y no de otras, y la adopción de ciertas formas de organización de la actividad conjunta y no de otras, por parte de sus usuarios. Y en segundo lugar, porque entornos y objetos de aprendizaje incluyen igualmente restricciones $\mathrm{y}$ potencialidades instruccionales (una cierta "inter-actividad pedagógica potencial"), que actúan también de modo similar. En último término, por ello, entornos virtuales y objetos de aprendizaje dificultan, permiten o promueven ciertas formas de enseñar y aprender, y devienen por ello tributarios de determinados modelos didácticos o instruccionales. Otra cosa es que esos modelos se hagan explícitos, o incluso que formen parte de las preocupaciones centrales de quienes los diseñan o los difunden. Como señala Wiley (2000):

"Software vendors and standard bodies describe their learning object related work as being "instructional theory neutral". Were this the case all would be well in learning object land. Problematically, a more accurate description of their products in "instructional theory agnostic", or in other words, "we dont'know if you're employing an instructional theory or not, and we don't care."”

Aprender y enseñar en entornos virtuales: Actividad conjunta, ayuda pedagógica y construcción del conocimiento. Javier Onrubia 
En no pocos casos, la concepción implícita de los procesos de enseñanza y aprendizaje que puede identificarse como subyacente a determinadas propuestas de diseño de entornos y objetos de aprendizaje es, en último término, una concepción en buena medida tributaria de modelos transmisivos y lineales de enseñanza, que otorgan escasa importancia tanto a los procesos de construcción del aprendiz y a la consideración de lo que el aprendiz aporta a su proceso de aprendizaje como a los procesos sociales de ayuda y soporte y a la consideración de lo que el profesor aporta a la orientación y "andamiaje" de esos procesos. En este sentido, algunos de los modelos de e-learning actualmente existentes, centrados fundamentalmente en la provisión y distribución de contenidos cerrados y estandarizados, resultan difícilmente compatibles con una visión de los procesos virtuales de enseñanza y aprendizaje como la que hemos esbozado. En primer lugar, porque presuponen una correspondencia lineal entre lo que se enseña y lo que se aprende, ignorando el papel de la actividad mental constructiva del alumno y de la actividad conjunta entre profesor y alumno, y entre alumnos, en el aprendizaje virtual. En segundo lugar, porque responden, en muchos casos, a una lógica que apuesta por la reducción, en ocasiones drástica, del papel de ayuda ejercido por el profesor, claramente contradictoria con el principio de ajuste de la ayuda y las características y requerimientos que plantea una ayuda ajustada. Y en tercer lugar, porque ignoran en buena medida, al menos en la práctica, la diferencia entre diseño y uso, entre lo que el diseño tecno-pedagógico del proceso de enseñanza y aprendizaje virtual plantea, y lo que los participantes realmente acaban haciendo en el proceso de desarrollo de ese diseño y en el uso concreto y situado de las herramientas, materiales y recursos que el diseño provee.

Frente a este tipo de planteamientos, resulta más coherente, desde una perspectiva socio-constructivista como la que hemos planteado, apostar por un modelo de diseño de entornos y objetos virtuales de aprendizaje que no se centre únicamente en la provisión y distribución de contenidos estandarizados, sino que preste especial atención a la creación de contextos que faciliten y promuevan las condiciones para que el profesor pueda ofrecer una ayuda ajustada a los aprendices, y pueda desarrollar con éxito los ajustes interaccionales que constituyen, hasta donde sabemos actualmente, el núcleo de los procesos y mecanismos de influencia educativa que concretan esa ayuda ajustada. En este modelo, la misión de las TIC y de los recursos tecnológicos virtuales no es reducir o eliminar el papel del profesor, sino por el contrario, amplificar y "empoderar" la "presencia docente". Ello supone primar aquellos recursos y usos de las TIC que permiten, precisamente, que el profesor pueda seguir de manera continuada el proceso de aprendizaje del alumno y ofrecer ayudas dinámicas, sensibles y contingentes, a ese proceso. Desde este planteamiento, entre las formas de utilización docente de las TIC más interesantes educativamente y que presentan un mayor valor añadido destacan, específicamente, aquellas que aprovechan y explotan en mayor medida los rasgos de las TIC como sistema semiótico de comunicación y representación que permiten al profesor ayudar más y mejor a los alumnos.

Aprender y enseñar en entornos virtuales: Actividad conjunta, ayuda pedagógica y construcción del conocimiento. Javier Onrubia 
En esta lógica, el punto de partida de las tareas de diseño no es únicamente el contenido a transmitir, sino también, y fundamentalmente, las formas de organización de la actividad conjunta que se pretende que aprendices y profesor desarrollen. Obviamente, ello no quiere decir que los contenidos no sean importantes, ni que no deban diseñarse cuidadosamente, y tampoco niega que la producción y difusión de contenidos y materiales virtuales de calidad sea un elemento fundamental para la calidad de los procesos virtuales de enseñanza y aprendizaje. Sí que supone una llamada de atención, sin embargo, sobre el hecho de que el diseño de contenidos es sólo una parte del problema más amplio del diseño de contextos y procesos virtuales de enseñanza y aprendizaje, y que en una perspectiva como la que hemos apuntado, es en la actividad conjunta entre profesores y alumnos en esos contextos y procesos donde acaba concretándose el uso real de esos contenidos y materiales, y donde acaba jugándose y decidiéndose en última instancia lo que los alumnos pueden aprender.

En un modelo de diseño como el que estamos proponiendo, adquiere particular relevancia e interés la inclusión de herramientas tecnológicas y recursos instruccionales dirigidos a apoyar virtualmente las formas de ayuda y mediación social implicadas en el ofrecimiento por parte del profesor de ayudas ajustadas. Por ejemplo, en el entorno "Knowledge Forum", los alumnos disponen de herramientas tecnológicas que "andamian" su proceso de generación de ideas, o que les facilitan la construcción de conocimiento a partir de las ideas y propuestas de otros (véase Scardamalia, 2004, para una descripción más detallada). Estas herramientas amplifican y potencian las ayudas sociales típicamente ofrecidas por el profesor en su papel de mediador del proceso de construcción colectiva del conocimiento. Coherentemente con la distinción entre diseño y uso que hemos reiterado, el valor de estas herramientas, desde nuestra perspectiva, no estriba en que puedan llegar a sustituir o hacer innecesaria la ayuda del profesor, sino en que aumentan y amplifican, gracias a las posibilidades tecnológicas, la presencia docente en el proceso de aprendizaje de los alumnos, creando un contexto que promueve en los alumnos ciertos tipos de actuaciones y formas de aprender que potencian la comprensión y elaboración significativa de conocimiento, así como ciertas formas de organización de la actividad conjunta centradas en el seguimiento y apoyo del profesor a esa comprensión y elaboración significativa.

Del mismo modo, un modelo de diseño como el que estamos planteando permite incorporar algunos elementos adicionales en el planteamiento de problemas clásicos en relación con el diseño de objetos de aprendizaje como la reusabilidad o la granularidad. Así, y por lo que hace referencia al primero de ellos, la perspectiva que estamos dibujando sugiere que la flexibilidad y la apertura de los objetos de aprendizaje pueden ser elementos clave para su reusabilidad: en efecto, la posibilidad de reutilizar un objeto de aprendizaje no tiene que ver, desde esta perspectiva, únicamente con su tamaño o sus características técnicas, sino también con el grado en que un determinado profesor pueda modificarlo, diversificarlo y adaptarlo desde el punto de vista pedagógico o instruccional a las características concretas de los alumnos, situaciones y contextos en que va a emplearlo. En cuanto al segundo, el problema de la granularidad se plantea

Aprender y enseñar en entornos virtuales: Actividad conjunta, ayuda pedagógica y construcción del conocimiento. Javier Onrubia

Página 9 de 14 
necesariamente, desde esta perspectiva, como una cuestión esencialmente educativa y relacionada con los procesos de planificación de la enseñanza: la decisión sobre el "grano" más adecuado para los objetos de aprendizaje debe considerar, en cualquier caso y eventualmente entre otras variables, la cuestión de qué tipo de "unidades" básicas de planificación de sus procesos virtuales de enseñanza y aprendizaje usan y requieren los profesores, y de si esas unidades son de la misma naturaleza y dimensión en distintos contextos de uso.

Finalmente, y ya para cerrar este punto, la importancia decisiva del contexto y las situaciones y prácticas de uso para la concreción de la efectividad y calidad de los entornos y objetos de enseñanza y aprendizaje que estamos postulando tiene también implicaciones importantes para el proceso mismo de realización de las tareas de diseño de tales entornos y objetos. Ello supone pasar de una concepción del diseño de entornos y objetos virtuales de enseñanza y aprendizaje como tarea exclusivamente técnica y completamente previa a la implementación en contextos reales a su caracterización como una tarea práctica y que supone una interrelación constante y continuada entre diseño y uso. Del mismo modo, ello supone también pasar de una concepción en que diseñadores y usuarios se encuentran radicalmente separados a una concepción basada en la comunicación y la colaboración mutua entre unos y otros. Determinadas comunidades de desarrolladores y usuarios de software libre educativo (por ejemplo, del tipo de comunidad establecida en torno a Moodle) ilustran adecuadamente esta última concepción y algunas de sus potencialidades.

\section{Algunas implicaciones para la evaluación de entornos y objetos virtuales de enseñanza y aprendizaje}

La importancia atribuida desde el planteamiento teórico propuesto a la actividad conjunta entre profesor y alumnos y los procesos y mecanismos de ayuda educativa en los procesos virtuales de enseñanza y aprendizaje tiene también implicaciones directas desde el punto de vista de la valoración de la calidad de los entornos y objetos virtuales de enseñanza y aprendizaje, y de su evaluación. Por un lado, supone situar las formas de organización de la actividad conjunta como foco fundamental de esa evaluación, y los procesos y mecanismos de ajuste como objeto central de la misma. Desde esta perspectiva, en efecto, la calidad de un entorno virtual de enseñanza y aprendizaje no está tanto en las herramientas técnicas de que dispone, en los materiales que incluye o en las actividades de aprendizaje que plantea a los alumnos considerados en sí mismos, cuanto en la manera en que esas herramientas, materiales y actividades se combinan y se ponen en juego para promover que alumnos y profesores se impliquen en unas u otras formas de actividad conjunta, y en la manera en que esas formas de actividad se organizan, combinan, secuencian y evolucionan a lo largo del proceso de enseñanza y aprendizaje, ofreciendo al profesor más o menos posibilidades de ajustar la ayuda a los alumnos.

Aprender y enseñar en entornos virtuales: Actividad conjunta, ayuda pedagógica y construcción del conocimiento. Javier Onrubia 
Por otro lado, las peculiares restricciones que afectan a la actividad conjunta en entornos virtuales llevan a la necesidad de distinguir diversos planos o niveles en el proceso de evaluación, particularmente, el plano de lo que hemos denominado "diseño tecnopedagógico", por un lado, y el plano de lo que hemos denominado "interactividad real", es decir, de la estructura de la actividad conjunta efectivamente desplegada por los participantes en el desarrollo real del proceso de enseñanza y aprendizaje, por otro. En el primer caso, el objeto de evaluación. En el primero de estos planos, y de acuerdo con el foco de evaluación que hemos propuesto, el interés fundamental se situaría en valorar cómo las características y herramientas tecnológicas del entorno, y las características del diseño instruccional previsto, prohiben, dificultan, permiten, promueven u obligan a los participantes a implicarse en determinadas formas de organización de la actividad conjunta. En el segundo, y en el mismo sentido, el interés se centraría en valorar el uso efectivo de las herramientas disponibles y la concreción que los participantes hacen del diseño previsto, y la estructura de la actividad conjunta real en que, a partir de todo ello, se implican los participantes. Para cada uno de estos planos es posible, a su vez, delimitar un conjunto de dimensiones y subdimensiones específicas que permitan operacionalizar esos distintos niveles de evaluación (Mauri et al., 2005).

La distinción entre el plano del diseño tecno-pedagógico y el plano de su concreción efectiva en la actividad conjunta real de profesor y alumnos comporta, también, la necesidad de incorporar múltiples puntos de vista y fuentes al proceso de evaluación. Diseñadores tecnológicos, diseñadores instruccionales, elaboradores de materiales y especialistas en el contenido objeto de enseñanza y aprendizaje, profesores y alumnos son, por citar sólo los más evidentes, algunos de los agentes a los que es necesario tomar en consideración para poder disponer de esa pluralidad de puntos de vista y fuentes (Barberà et al., 2004). A su vez, la incorporación de este amplio conjunto de planos, dimensiones, puntos de vista y fuentes de evaluación conlleva la necesidad de diversificar y multiplicar los instrumentos metodológicos de evaluación. A este respecto, cabe destacar la importancia que, en un modelo de evaluación como el que estamos bosquejando, tienen las técnicas de recogida de información que permiten acceder "en vivo" al desarrollo real de los procesos virtuales de enseñanza y aprendizaje, ofreciendo información concreta sobre cómo profesor y alumnos llevan a cabo, articulan e interrelacionan sus actuaciones en torno a las tareas y actividades que vertebran el proceso virtual de enseñanza y aprendizaje.

\section{A modo de conclusión}

Como señalábamos al inicio, la relación entre las TIC y la mejora de las prácticas educativas dista de ser lineal o sencilla. Las TIC abren, sin duda, por sus propias características, nuevas posibilidades de innovación y mejora de los procesos formales de enseñanza y aprendizaje, pero la mera incorporación de herramientas tecnológicas a las prácticas educativas no garantiza en modo alguno que esa mejora se produzca realmente. De hecho, existen indicios de que lo que ocurre, al menos en determinadas ocasiones, es exactamente lo contrario: que la introducción de las TIC en las prácticas

Aprender y enseñar en entornos virtuales: Actividad conjunta, ayuda pedagógica y construcción del conocimiento. Javier Onrubia

Página 11 de 14 
educativas sirve más para reforzar los modelos dominantes y ya establecidos de enseñanza y aprendizaje que para modificarlos (véanse, por ejemplo, los resultados del informe sobre el uso de Internet en las escuelas catalanas elaborado recientemente por investigadores de la Universitat Oberta de Catalunya (IN3-UOC, 2004)).

Por ello, quisiéramos cerrar nuestra exposición haciendo una apelación a la importancia de mantener y avivar la reflexión sobre los objetivos y criterios que deben guiar la incorporación de las TIC a las prácticas educativas, así como también a la importancia de la investigación empírica sobre los resultados y aportaciones de dicha incorporación para la mejora de la calidad de la enseñanza. En el primer caso, entendemos que es extremadamente importante que esa reflexión priorice las consideraciones específicamente educativas (frente, por ejemplo, a las de carácter tecnológico o económico), e incluya un amplio debate teórico sobre los modelos y principios psicológicos y educativos que deban guiar la incorporación de las TIC a los procesos de enseñanza y aprendizaje. Las nociones e ideas que hemos propuesto tienen, sin duda, la pretensión de alimentar y contribuir a este debate en el ámbito específico de los procesos, entornos y objetos virtuales de enseñanza y aprendizaje; un ámbito en el que las posiciones constructivistas han ido reclamando cada vez más protagonismo en los últimos tiempos (p.e. Bannan-Ritland, Dabbagh y Murphy, 2000). En cuanto a la investigación empírica sobre los resultados y aportaciones de las TIC para la mejora de la calidad de la enseñanza, somos partidarios de una estrategia que combine de manera sistemática innovación docente, investigación educativa y evaluación de los efectos de la innovación. El diseño, desarrollo y evaluación de "experimentos formativos" de utilización educativa de las TIC, dirigidos a la mejora de las prácticas docentes y los procesos de aprendizaje de los alumnos en contextos y situaciones concretos, puede ser, a nuestro juicio, una de las vías más prometedoras para la concreción de una estrategia de estas características.

Presentación del artículo: Enero de 2005 Fecha de aprobación: 5 de Febrero de 2005 Fecha de publicación (en su primera edición): 20 de Febrero de 2005

Onrubia, J. (2016). Aprender y enseñar en entornos virtuales: Actividad conjunta, ayuda pedagógica y construcción del conocimiento. RED. Revista de Educación a Distancia. 50(3). Consultado el (dd/mm/aaaa) en http://www.um.es/ead/red/50

\section{Referencias}

Bannan-Ritland, B., Dabbagh, N. y Murphy, K. (2000). Learning Object Systems as Constructivist Learning Environments: Related Assumptions, Theories and Applications. En D. A. Wiley (Ed.), The Instructional Use of Learning Objects: Online Version. Consultado el 24 de enero de 2005 en: http://reusability.org/read/chapters/wiley.doc 
Barberà, E., Badia, A., Colomina, R., Coll, C., Espasa, A, Gispert, I. de, Lafuente, M., Mayordomo, R., Mauri, T., Naranjo, M., Onrubia, J., Remesal, A., Rochera, M. J., Segués, T., Sigalés, C. (2004). Pautas para el análisis de la intervención en

entornos de aprendizaje virtual: dimensiones relevantes e instrumentos de evaluación [documento de proyecto en línea]. IN3:UOC. (Discussion Paper Series: DP04-002) [Consultado el 24 de enero de 2005 en: <http://www.uoc.edu/in3/dt/esp/barbera0704.html>

Coll, C. (2001). Constructivismo y educación: la concepción constructivista de la enseñanza y el aprendizaje. En C. Coll, J. Palacios y A. Marchesi (Comps.), Desarrollo psicológico y educación. 2. Psicología de la educación escolar (pp. 157-188). Madrid: Alianza.

Coll, C. (en prensa). Psicología de la educación y prácticas educativas mediadas por las tecnologías de la información y la comunicación. Una mirada constructivista. Sinéctica.

Coll, C. \& Martí, E. (2001). La educación escolar ante las nuevas tecnologías de la información y la comunicación. En C. Coll, J. Palacios y A. Marchesi (Comps.), Desarrollo psicológico y educación. 2. Psicología de la educación escolar (pp. 623-651). Madrid: Alianza.

IN3-UOC (2004). L'escola a la societat xarxa. Internet en l'àmbit educatiu no universitari (Informe de recerca. Volum I). Consultado el 24 de enero de 2005 en 〈http://www.uoc.edu/in3/pic/cat/pdf/PIC_escoles_0.pdf>

Mauri, T., Onrubia, J., Coll, C. y Colomina, R. (2005 - pendiente de publicación). La calidad de los contenidos educativos reutilizables: diseño, usabilidad y prácticas de uso. RED. Revista de educación a distancia.

Scardamalia, M. (2004) CSILE/Knowledge Forum. En Education and technology: An encyclopedia (pp. 183-192). Santa Barbara: ABC-CLIO

Wiley, D. A. (2000). Connecting learning objects to instructional design theory: A definition, a metaphor, and a taxonomy. En D. A. Wiley (Ed.), The Instructional Use of Learning Objects: Online Version. Consultado el 24 de enero de 2005 en: http://reusability.org/read/chapters/wiley.doc

\footnotetext{
${ }^{\text {i }}$ El marco que presentaremos se inspira directamente en algunos de los conceptos e ideas principales propuestos por la denominada "concepción constructivista del aprendizaje escolar y de la enseñanza" (Coll, 2001) y en la visión que desde esta
} 
concepción se plantea de los procesos de enseñanza y aprendizaje mediados por las tecnologías de la información y la comunicación (Coll y Martí, 2001; Coll, en prensa). 